\title{
THE RIGHT VENTRICLE AND THE SMALL PULMONARY ARTERIES IN COALWORKERS
}

\author{
BY \\ ARTHUR J. THOMAS AND W. R. L. JAMES \\ From Llandough Hospital (United Cardiff Hospitals) and the Department of Pathology, \\ Welsh National School of Medicine
}

Received November 27, 1957

In previous work (Thomas, 1951; James and Thomas, 1956), we have been made aware of some problems of pulmonary heart disease that needed further elucidation. Further consideration of the method of assessment of right ventricular hypertrophy and its relation to emphysema and pneumoconiosis when present, separately or together, was needed. Jaffe (1934), referred to the lesion of pneumoconiosis as including obliterative changes in the branches of the pulmonary artery. Other workers (Gough, 1952; Wells, 1954; James and Thomas, 1956), have referred to the presence of arterial lesions in the vessels of the lungs in pneumoconiosis. It seemed important to examine the relationship of these vascular changes to the right ventricular hypertrophy. The nature of the changes in the vessels is also in question. Pulmonary thrombosis has been regarded as a relatively rare happening, but we have found that in pulmonary heart disease, especially in coalworkers, it is much commoner than is thought. Other workers have seen differing kinds of arterial lesions in the lungs in other forms of pulmonary hypertension (McKeown, 1952; Wade and Ball, 1957), and it seemed important to try and assess the nature of the change in pneumoconiosis. Heart failure and lung disease has been reviewed by Hecht (1956), and from this review it is clear that forms of heart failure can differ in different pulmonary diseases.

\section{MATERIAL AND MethOD}

The lungs and hearts from 71 necropsies on coalworkers were examined. The cases chosen were those likely to show right ventricular hypertrophy due to pulmonary disease.

The hearts were examined at necropsy and then fixed and re-examined by dissection so as to record the left and right ventricular weights. The ventricles were separated through the septum by the method of Hermann and Wilson (1922) and weighed. The free walls of the ventricles were then separated from the attached septal portions and the parts weighed separately. The thickness of the ventricular walls near the base of the heart had been measured in routine fashion at necropsy. There was thus available total heart weight, ventricular wall thickness as assessed at necropsy, ventricular weights including the share of the septum, free walls of both ventricles, and the separated right and left portions of the septum.

At necropsy one of us (W.R.L.J.) assumed responsibility for the preparation and examination of the lung specimens. One of the lungs was cut at that time and examined naked-eye before fixation. The other was perfused with formol-sodium acetate solution through the bronchus and allowed to fix in a distended state for one or more days. From each lung, blocks were taken for microscopic study. Some pieces were cut from areas of massive fibrosis and others from areas affected by simple pneumoconiosis only. The number of pieces of lung tissue examined varied from case to case, the minimum being 8 and the maximum 17. The sections obtained were stained with hæmalum and eosin, and studied microscopically with special reference to the state of the pulmonary arteries. From the lung that was perfused at necropsy, a whole lung section was made by the technique of Gough and Wentworth. 
In studying the microscopic preparations, attention was directed mainly to the following branches of the pulmonary arteries as classified by Brenner (1935): (1) muscular arteries with external diameters ranging from $100 \mu$ to $1000 \mu$, and (2) arterioles with external diameters usually less than $100 \mu$. One of us (W.R.L.J.) subjectively assessed the severity of the lesions in these vessels as slight, moderate, or severe, on the basis of the examination of all the microscopic preparations available in each case. The other (A.J.T.), independently studied the heart and the relationship between the findings is shown (Fig. 1).

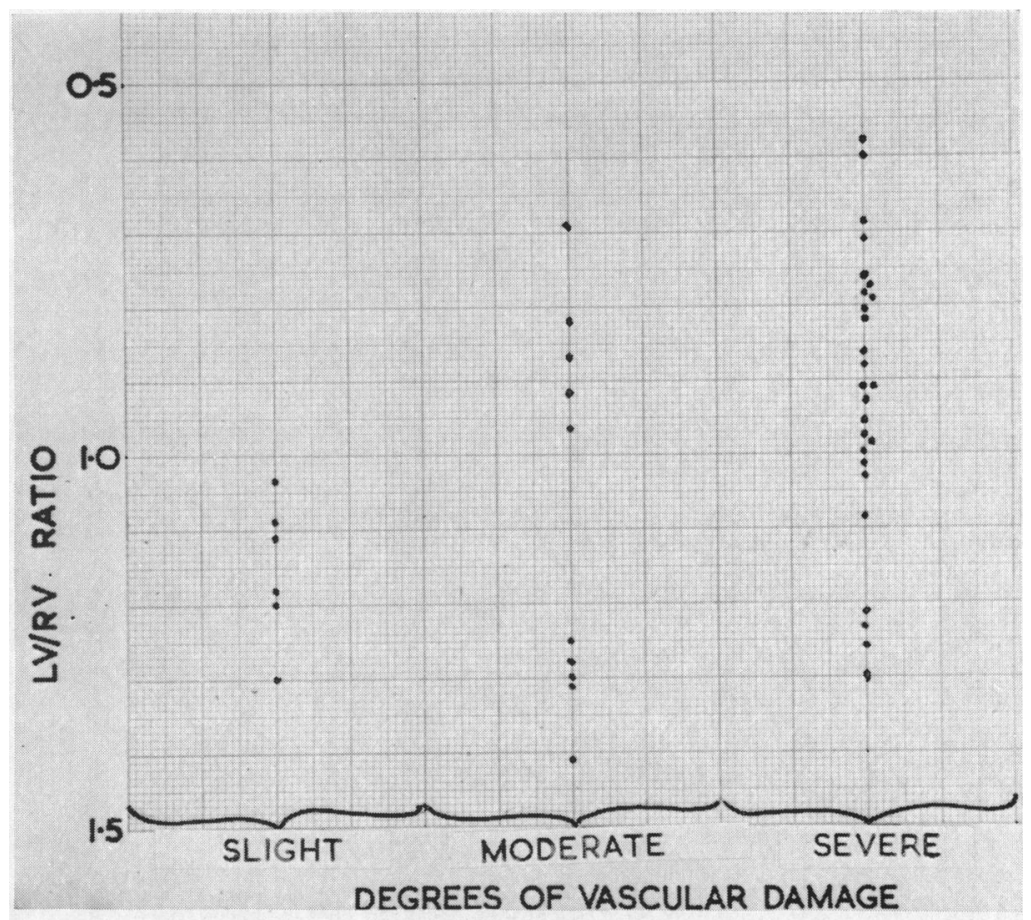

FIG. 1.-The relationship of the degrees of right ventricular hypertrophy and of severity of changes in the small pulmonary arteries.

As a result of the examination of the coronary arteries and the myocardium in detail it was necessary to discard from the pulmonary study 23 cases, because 22 had coronary heart disease of such severe degree as to affect decisions on cardiac pathology and one had aortic stenosis. This figure of 34 per cent of severe coronary heart disease is of the greatest interest, as we have pointed out elsewhere that on all occasions when we study coalworkers, we find a high incidence of coronary heart disease of this order. The detailed findings in the rejected cases will be described in a separate communication.

There remained 48 cases to be analysed in relation to the character and extent of the pulmonary disease and the degree of right ventricular hypertrophy.

\section{RESULTS}

Assessment of Ventricular Hypertrophy. The correlation of the LV/RV ratio of the dissected free ventricular walls with the LV/RV ratio of the ventricles when separated through the septum was close. The former is an easier method of detecting right ventricular hypertrophy or preponderance. 
In right ventricular hypertrophy we have noted large muscle columns arising from the right side of the septum and clearly part of the hypertrophy of the right side of the septum. It is clear that, when considering right ventricular hypertrophy alone, the total septal weight cannot be added to the weight of the left ventricle, for the error produced may be significant and may mask the slighter degrees of right ventricular preponderance, especially in small hearts (Fig. 2).

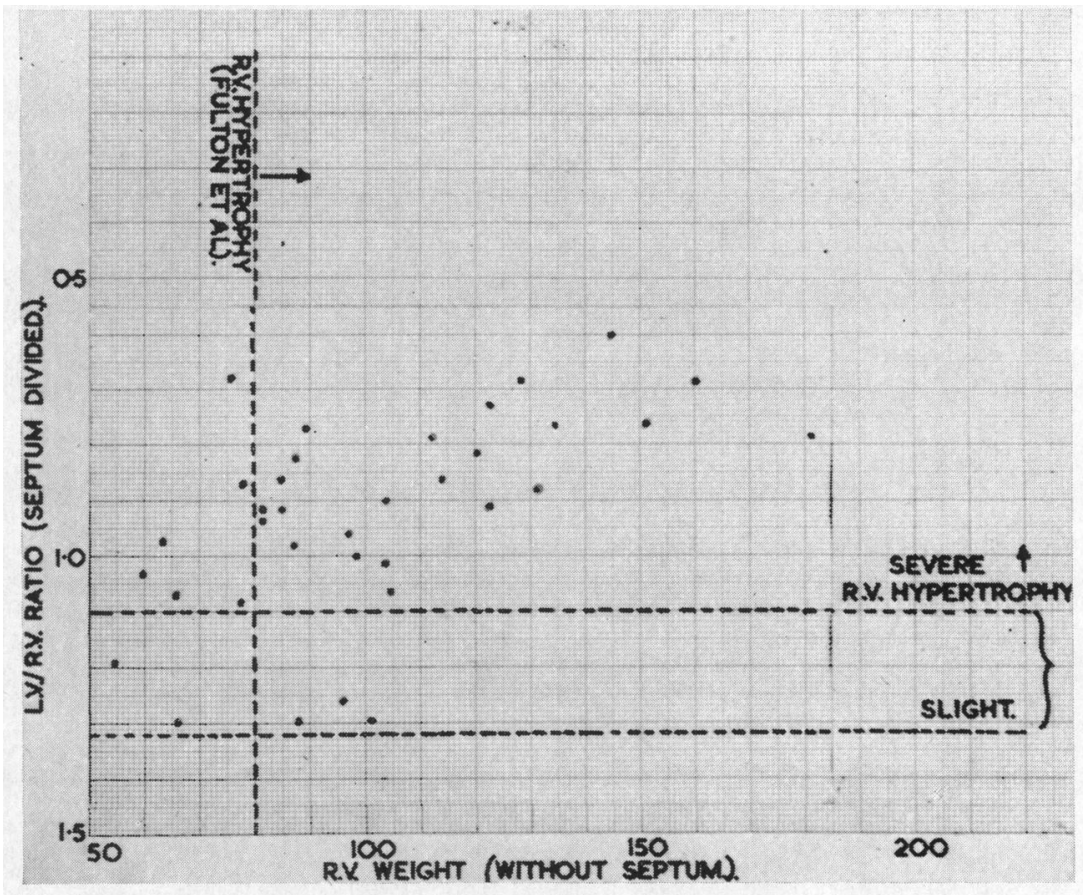

FIG. 2.-The correlation of the LV/RV ratio with the right ventricular weight as a criterion of hypertrophy. If a free right ventricular wall weight of over $80 \mathrm{~g}$. is required for hypertrophy, then right ventricular preponderance in some small hearts will not be detected.

When we considered the thicknesses of the right ventricular and left ventricular walls we found that there was good correlation with LV/RV ratios over the middle ranges of right ventricular hypertrophy, but in small hearts with right ventricular wall thicknesses of 3 or $4 \mathrm{~mm}$., the LV/RV ratio often showed right ventricular preponderance (Fig. 3), which would not be detected by simple measurement of right ventricular thickness. In several such cases the chronic venous congestion of heart failure was present. The right ventricular wall thickness of $5 \mathrm{~mm}$. is an equivocal measure and may or may not be a true indication of right ventricular hypertrophy (Fig. 3), but if it is above $5 \mathrm{~mm}$. it always correlates well with true hypertrophy.

The form of the hypertrophy is not consistent; in some cases trabecular hypertrophy is striking but in others there is a more compact hypertrophy of the whole wall. It may be that the degree of dilatation decides the prominence of the trabeculæ.

The Pulmonary Disease and Vascular Changes. In the areas of lung showing only simple pneumoconiosis normal vessels were the rule. On examining the sections prepared from areas of massive fibrosis the tissue was relatively avascular in comparison with the rest of the lung and almost all vessels that had survived were abnormal. No examples of frank vascular necrosis were found, but in most vessels there was distortion, degeneration, and evidence of reduction of the lumen by disease of the vessel wall or thrombosis. 


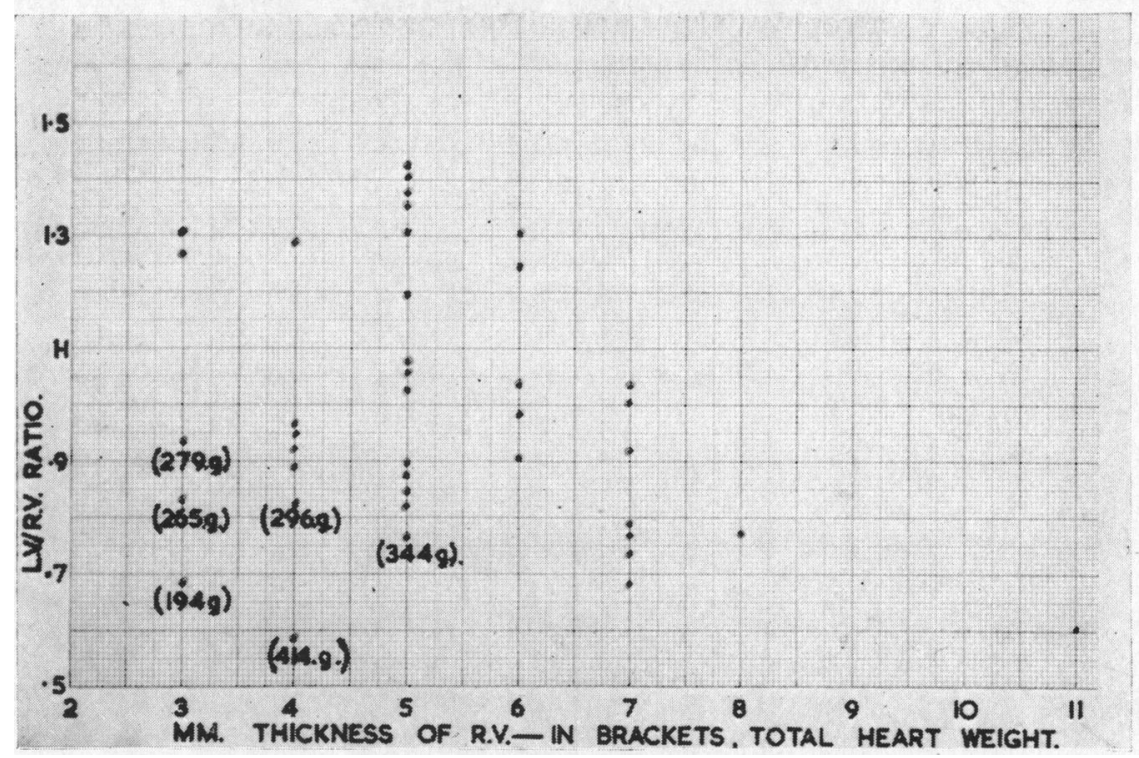

FIG. 3.- The distribution of right ventricular hypertrophy determined by LV/RV ratio and by thickness of right ventricular wall. It is noted that small hearts, and also one large but dilated heart, tend to have a relatively thin right ventricular wall.

In many vessels there was gross disproportion between the area of the vessel wall section and the area of the lumen, with fibrous distortion of the vessel wall (Fig. 4), and in many the wall was infiltrated with scanty plasma cells and lymphocytes and numerous dust-laden macrophages, and there were multiple lumina due to recanalized thrombi (Fig. 5). Sometimes compression of adjacent structures such as bronchi indicated that vascular swelling had occurred (Fig. 6).

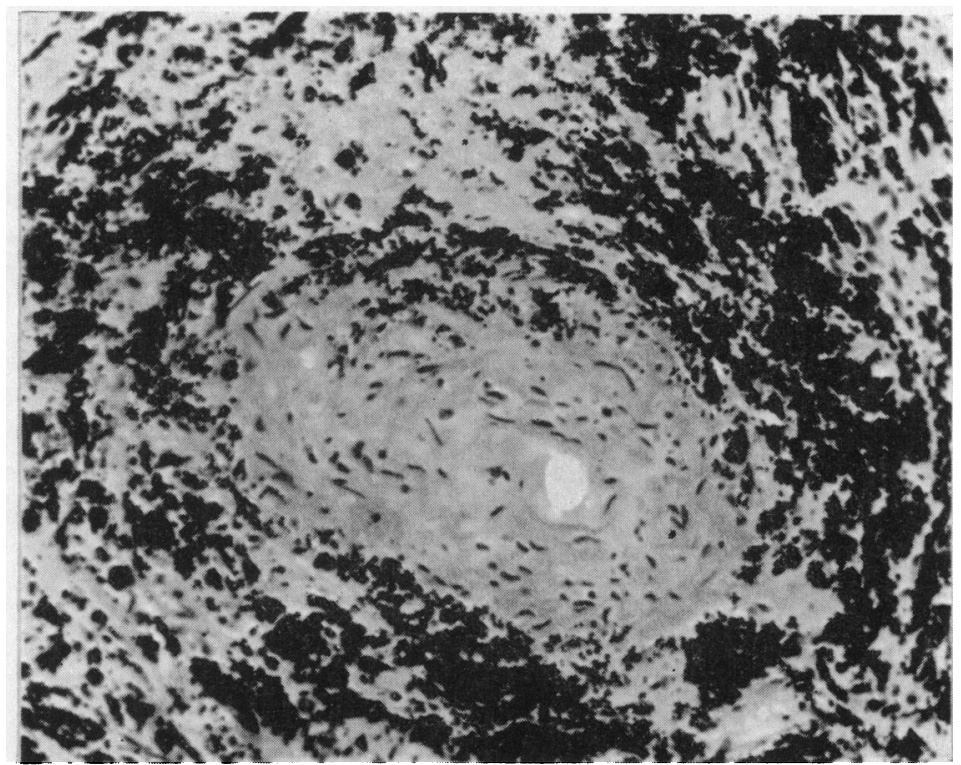

FIG. 4. - $(\times 180)$. A vessel of muscular artery size in an area of massive dust fibrosis. The wall is grossly fibrosed and thickened and few muscle cells have survived. (Age 48 years, LV/RV ratio, 0.79, R.V. weight 132 g.) 


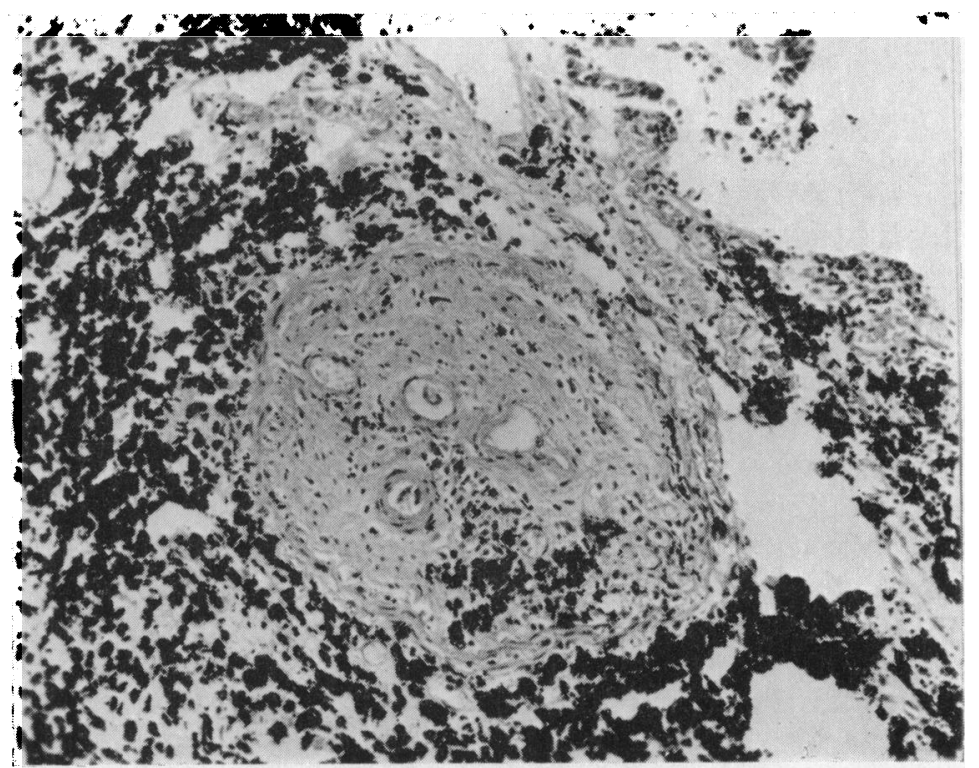

FIG. 5.- $(\times 120)$. The wall of the vessel is thick and consists mainly of fibrous tissue infiltrated by dust laden macrophages and chronic inflammatory cells. The four lumina indicate recanalization of old thrombus. (Age 52 years, $L V / R V$ ratio $0.91, R$.V. weight 112 g.)

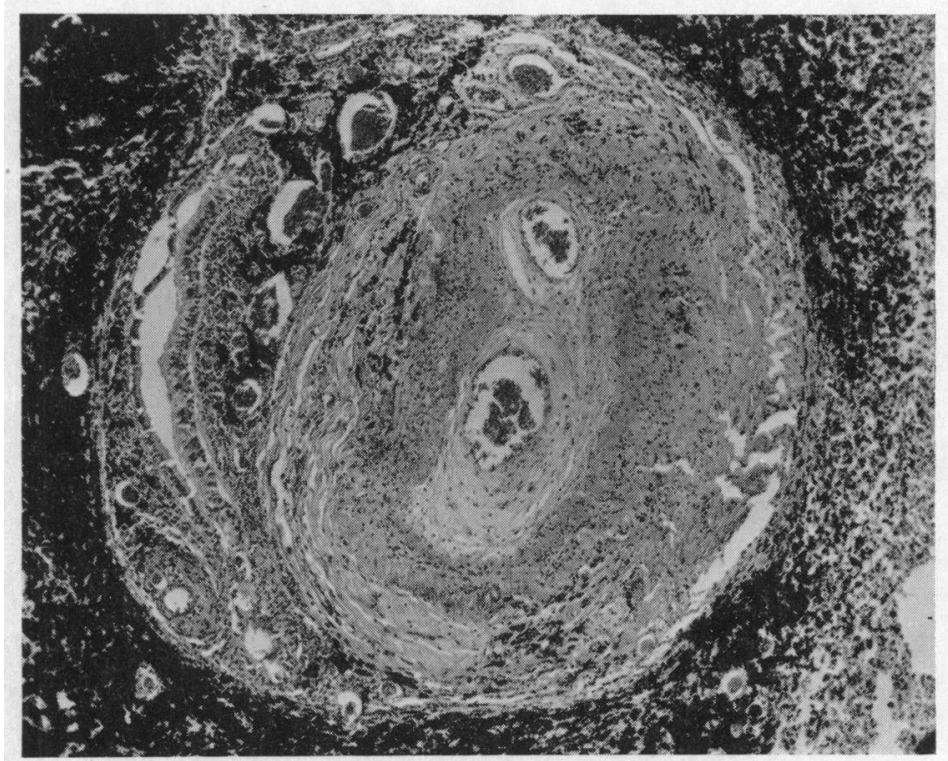

FIG. 6. $-(\times 50)$. The vessel wall is fibrosed and the lumen is reduced by an organized recanalized thrombus. The lumen of the adjacent bronchiole is reduced to a narrow slit, apparently as a result of the enlargement of the artery. The electrocardiogram of this case shows the right ventricular hypertrophy. (Age 35 years, LV/RV ratio 0.6, R.V. weight $170 \mathrm{~g}$.) 
In 8 of the 48 cases examined the vascular lesions were graded as slight, in 12 as moderate, and in 28 as severe. The relationship between the severity of the lesions and the LV/RV ratio is shown (Fig. 1).

The Right Ventricular Changes. An analysis based on the state of the right ventricle showed severe hypertrophy in 31 , slight hypertrophy in 11 , and none in 6 . The 31 with severe hypertrophy had an LV/RV ratio below 1.1. The mean age of the 31 patients was 62 years (35 to 73 years), the mean heart weight was $324 \mathrm{~g}$. (194-545 g.), and the mean weight of the right ventricle was $127 \mathrm{~g}$. (range 70-220 g.).

Of the 31 with an $L V / R V$ ratio below $1 \cdot 1$, there were 9 whose right ventricular wall thickness was 3 or $4 \mathrm{~mm}$. and in 7 of them the hearts were small. In 4 of these, pulmonary tuberculosis had been demonstrated.

Another sub-division of great importance in the 31 cases was the group of 9 with progressive massive fibrosis and little or no other pulmonary disease. The mean age of the 9 patients was 52 years ( 35 to 63 years), the mean ratio of $L V / R V$ was $0 \cdot 88$, the right ventricular mean weight was $139 \mathrm{~g}$. (77-220 g.), and the right ventricular thickness was considerable in all except two, one of whom had tuberculosis.

When we tried to express the extent of the progressive massive fibrosis as a factor, using the sum of the maximum diameters in centimetres of the massive lesions in both lungs, we found a mean "PMF" factor of 19 in the cases of severe right ventricular hypertrophy. This would have been higher but for two instances with readings of ten. There was one unusual case without massive fibrosis, but with gross arterial changes in many small fibrous lesions resulting in hypertrophy.

There was pulmonary thrombosis of macroscopic degree in 7 of the 31 cases and it was not all formed just before death. Histological evidence of thrombus organization was frequent in medium sized arteries. The occurrence of thrombosis seemed sometimes to have caused a recognizable clinical episode.

Chronic venous congestion of the liver and spleen was almost always present at necropsy, although not always recognized clinically. A palpable tender liver margin is a good early sign of pulmonary heart failure in these cases (Thomas, 1948). Electrocardiograms taken at a late stage of the illness always showed an indication of pulmonary heart disease, usually pulmonary $P$ waves, vertical heart pattern, and R-S complexes in V6. In many there was unequivocal evidence of right ventricular hypertrophy.

Slight Right Ventricular Hypertrophy. This group with LV/RV ratio $1 \cdot 1$ to $1 \cdot 3$ inclusive comprised 11 patients whose mean age was 69 years (range 51-81), an older group than those with severe right ventricular hypertrophy. The mean heart weight was $278 \mathrm{~g}$. (range 207-339 g.), and the mean right ventricular weight was $93 \mathrm{~g}$. (range 67-125 g.). So although they were a slightly older group with rather smaller hearts, they still had evidence of the presence of some right ventricular hypertrophy. It is proper to take this group by their LV/RV ratio because, when the thicknesses are taken, one finds that there are right ventricles of $3 \mathrm{~mm}$. and $4 \mathrm{~mm}$. in patients who had chronic venous congestion and died in right heart failure.

In those with slight right ventricular hypertrophy three had progressive massive fibrosis but no emphysema. In this group as a whole the vascular lesions were much less intense than in those with severe right ventricular hypertrophy.

Absence of Hypertrophy. There remained a small group of 6 cases with LV/RV ratio over $1 \cdot 3$ which was incompatible with isolated right ventricular hypertrophy, though this was expected at necropsy. Their mean age was 60 years, but ranged from 39 years to 81 years, and their mean heart weight was $325 \mathrm{~g}$. (235-455 g.). The average progressive massive fibrosis factor was 12 . 
The vascular lesions were slight in one, moderate in four, and severe in one. This last case should have shown right ventricular hypertrophy; one possible explanation for his not doing so was protection by a long period of reduced physical activity. Tuberculosis was present in two cases and rheumatoid arthritis in one.

\section{Discussion}

A study of the pulmonary blood vessels in coalworkers' lungs has led us to suggest, as have others previously (Jaffe, 1934; Gough, 1952), that the change in the medium and small arteries is responsible for the increased right ventricular work resulting in hypertrophy. The changes in the vessels are fibrosis and thickening of the muscular wall, with disappearance of the muscle fibres and reduction of the lumen due to intimal thickening or fibrosis. There is a recognizably high incidence of thrombosis in the large and medium arteries and this may well happen in the small vessels too.

This type of pulmonary disease is commonly a mixture of massive fibrosis, emphysema, and "chronic bronchitis". By identifying 9 cases of massive fibrosis without emphysema but with severe arterial lesions and right ventricular hypertrophy, we have shown the part that such fibrosis can play in producing right ventricular hypertrophy. Also it becomes evident that smaller areas of lung fibrosis, with lesser degrees of arterial change, are as a rule accompanied by slighter grades of right ventricular hypertrophy.

The total heart weight is often low, sometimes in association with recognized tuberculosis, and in such cases it becomes necessary to weigh the right and left ventricles by some method, to identify the relative right ventricular hypertrophy. The right ventricular wall thickness can be misleading in these circumstances. There is usually ample evidence of chronic venous congestion in the liver and spleen to support the diagnosis of pulmonary heart disease.

Some points in this study suggest that emphysema and bronchitis hasten the development of the pulmonary heart disease and certainly recurrent exacerbation of infection does so. Of equal interest is some evidence that reduction of physical activity over a long period delays the final episode of heart failure, and may also slow the rate of hypertrophy of the right ventricle. Conversely, persistence in physical activity, as in the younger age group, increases the rate of development and degree of hypertrophy of the right ventricle. This can be explained by the presence of the inelastic or obstructive pulmonary vascular state, as there is a stage when the pulmonary artery pressure is normal at rest, but is raised progressively on effort. This causes an increase in right ventricular work and we think that, once the right ventricle has to work harder to maintain the pulmonary flow on slight exercise, then an irreversible sequence of events has started.

\section{SUMMARY}

The lungs and hearts from 71 necropsies in coalworkers have been examined and, after rejecting 23 cases because of coronary heart disease in 22 and aortic stenosis in 1, 48 have been analysed in relation to the right ventricular hypertrophy and the extent of changes in the smaller pulmonary arteries. The lungs were studied macroscopically and by microscopic preparations.

It was found that the LV/RV ratio obtained by full dissection correlated well with the LV/RV ratio obtained by weighing the free walls taken off close to the septum. Thicknesses of the right ventricle above $5 \mathrm{~mm}$. correlated well with right ventricular hypertrophy, but in some cases values of $3 \mathrm{~mm}$. and $4 \mathrm{~mm}$. were recorded in hearts shown by dissection to have right ventricular preponderance. The latter were usually small hearts, but the accompanying severe chronic venous congestion of liver and spleen supported their classification as pulmonary heart disease.

Lungs showing simple pneumoconiosis as a rule had normal vessels. Areas of progressive massive fibrosis showed relatively avascular regions with all surviving vessels around them abnormal.

Severe vascular lesions were present with moderate or severe right ventricular hypertrophy. In $2 \mathbf{F}$ 
9 cases massive fibrosis with vascular change was the only reason found for right ventricular hypertrophy. The general correlation suggests that the changes in the smaller pulmonary arteries and the arterioles are responsible for the right ventricular hypertrophy.

We are grateful to Professor J. Gough of the Department of Pathology, Welsh National School of Medicine for his help and advice, and to Dr. J. C. Gilson, Director, and Dr. Rivers, Pathologist, Pneumoconiosis Research Unit (MRC) for access to records and permission to study their cases. We would like to thank Mr. Leighton Williams, A.I.B.P., and Mr. Napper for the photographic illustrations.

\section{REFERENCES}

Brenner, O. (1935). Arch. intern. Med., 56, 976.

Fulton, R. M., Hutchinson, E. C., and Jones, A. M. (1952). Brit. Heart J., 14, 413.

Gough, J. (1952). A paper read before the Fourth Conference of the McIntyre Research Foundation on Silicosis, Noranda, Quebec, January, 1952.

Hecht, H. H. (1956). Circulation, 14, 265.

Hermann, G. R., and Wilson, F. N. (1922). Heart, 9, 91.

Jaffe, R. H. (1934). Illinois med. J., 66, 431.

James, W. R. L., and Thomas, A. J. (1956). Brit. J. industr. Med., 13, 24.

McKeown, F. (1952). Brit. Heart J., 14, 25.

Thomas, A. J. (1948). Brit. Heart J., 10, 282. (1951). Brit. Heart J., 13, 1.

Wade, G., and Ball, J. (1957). Quart. J. Med., N.S., 26, 83.

Wells, A. L. (1954). Brit. Heart J., 16, 74. 